\title{
Communication Modulation Signal Recognition Algorithm Based on Entropy Cloud Characteristics
}

\author{
Yibing $\mathrm{Li}^{\text {a }}$, Jie Chen ${ }^{\mathrm{a}}$, Dandan Liu ${ }^{\mathrm{b}}$ and Jingchao $\mathrm{Li}^{\mathrm{a}}$ \\ a:College of Information and Communication Engineering Harbin Engineering \\ University Harbin 150001, China \\ b: College of Electrical and Control Engineering, Heilongjiang University of \\ Science and Technology, Harbin 150001, China \\ Sandra@hrbeu.edu.cn
}

\begin{abstract}
Communication modulation signal recognition, as an emerging technology, has been widely used in the field of communication reconnaissance. It is generally known that the characteristics of communication modulation signals under low SNR environment is difficult to extract. To solve this problem, a novel modulation signal feature extraction algorithm based on entropy cloud characteristics is put forward in three steps by this paper. Firstly, it extracts entropy characteristics of the modification signal, which introduce the exponential entropy to construct two-dimensional feature entropy with shannon and exponential entropy for a better signal recognition performance. Then, it extracts cloud digital characteristics of information entropy to build three-dimensional feature, which can depict the modulation type characteristics of the signal. Finally, it uses grey correlation classifier for signal identification. By means of simulation, it can be seen that the new algorithm has overcome the defection that signal characteristics are unstable and difficult to extract under low SNR environment in the traditional method. So the new algorithm is available and effective for the signal identification under low SNR environment, thus achieves the goal of the signal classification.
\end{abstract}

Keywords: Signal recognition; Feature extraction; Entropy characteristics; Cloud model; Low SNR identification

\section{Introduction}

Recently, with the rapid development of computer technology, artificial intelligence, pattern recognition and signal processing, Communication modulation signal recognition, as an emerging technology, has drawn attention and been widely used in the field of communication reconnaissance. Modulation signal recognition is a key step between signal detection and signal demodulation [1-3], whose purpose is to judge the signal modulation types and estimate the corresponding modulation parameters through the received signal processing without other prior knowledge. It has been applied in two main aspects: one is the software radio system, which can guarantee the interconnection and interoperability of disparate communication systems, the other one is the electronic warfare system, which provides the basis for intercepting information and choosing the optimal jamming type.

There are many modulation signal recognition algorithms at present, such as the higher-order cumulant recognition algorithm [4], the entropy feature complexity recognition algorithm [5-6], the cyclic spectrum recognition algorithm [7], the higherorder spectrum feature recognition algorithm [8], the recognition algorithm based on fractal box-counting dimension [9-10], and the recognition algorithm based on constellation diagram[11], etc. How to achieve higher recognition rate with smaller 
computational complexity and less computing time under low SNR is the key to feature extraction.

Literature [12] proposes a digital modulation signal identification algorithm based on instantaneous characteristic parameters. It just uses five relatively simple instantaneous characteristic parameters to identify the modulation signal, so its computation is simple. At the same time, the recognition effect of the algorithm can be improved by using the wavelet filter to denoise the characteristic parameters. When the requirements for signal recognition rate is not high, this recognition algorithm is suitable for application. According to the amplitude, frequency and phase characteristics of the signal sample, Literature [13] uses the wavelet transform to identify different modulation signals in Gaussian channel environment. However, this algorithm has some limitations, which is only suitable for Gaussian noise environment with higher SNR. Literature [14] proposes a characteristics parameter identification algorithm based on the first-order statistical moments of the signal. It has the advantages of small computational complexity and good stability, and recognition rate can reach $97 \%$ even if the SNR is $-7 \mathrm{~dB}$. But the algorithm can only identify limited types of signals.

According to the analyses above, this paper puts forward a novel communication modulation signal recognition algorithm based on entropy cloud characteristics. On the basis of extracted signal entropy feature, we can extract the cloud model characteristics of signal entropy feature to build three-dimensional feature. Cloud characteristics extraction provides a good theoretical basis for the signal recognition under low SNR.

\section{Entropy Feature}

\subsection{Basic Definition}

Entropy is a very important concept in information theory, it is a metric of information uncertainty. We define the set of events as $X$, and use n-dimensional probability vector $p=\left(p_{1}, p_{2}, \cdots p_{n}\right)$ to represent the probability set of each event, which should meet:

$$
0 \leq p_{i} \leq 1
$$

and

$$
\sum_{i=1}^{n} p_{i}=1
$$

The entropy $H(p)$ can be defined as:

$$
H(p)=H\left(p_{1}, p_{2}, \cdots p_{n}\right)=\sum_{i=1}^{n} p_{i} \log \frac{1}{p_{i}}=-\sum_{i=1}^{n} p_{i} \log p_{i}
$$

As a result, $H(p)$ can be regarded as a function of the n-dimensional probability vector $p=\left(p_{1}, p_{2}, \cdots p_{n}\right)$, so we define it as the entropy function.

\subsection{Properties}

By the definition of the entropy function, $H(p)$ has the following properties:

1.The symmetry

When the order of each components $p_{1}, p_{2}, \cdots p_{n}$ are changed arbitrarily, the value of $H(p)$ remains the same, that is, the result of the entropy is only associated with the overall statistical properties of the set $X$.

2. The non-negativity

The value of the entropy function is a non-negative value, namely: 


\section{The certainty}

If there is a certain event in set $X$, the entropy value of $X$ must be zero.

4. The extreme

In the set $X$, when each event has the same probability, it gets the maximum entropy.

As: when $p_{1}=p_{2}=\cdots=p_{n}=\frac{1}{n}$,

$$
H\left(p_{1}, p_{2}, \cdots, p_{n}\right) \leq H\left(\frac{1}{n}, \frac{1}{n}, \cdots, \frac{1}{n}\right)=\log n
$$

\subsection{Two-Dimensional Feature Entropy}

Based on the definition of the shannon entropy, we introduced the definition of the exponential entropy to construct two-dimensional feature entropy for the better signal recognition. Assuming the probability of an event is $p_{i}$, then the amount of information can be defined as:

$$
\Delta I_{\exp }\left(p_{i}\right)=e^{1-p_{i}}
$$

According to the basic definition of the entropy, the exponential entropy $H_{\text {exp }}$ can be defined as:

$$
H_{\exp }=\sum_{i=1}^{n} p_{i} e^{1-p_{i}}
$$

From formula(6) and (7), it can be seen that compared with the traditional information $\Delta I\left(p_{i}\right)=\log \left(1 / p_{i}\right)$, the new definition $\Delta I_{\exp }\left(p_{i}\right)$ has the same meaning. In the domain of definition $[0,1], \Delta I_{\exp }\left(p_{i}\right)$ is monotony decrease function, and its range is $[1, e]$. If and only if the probability $p_{i}$ of all events are equal, $H_{\exp }$ will get the maximum value.

Entropy analysis algorithm uses the uncertainty of information for feature selection, and it doesn't need to know the size and distribution of the characteristic variables of the signal. At the same time, it has small amount of calculation, therefore, it is a relatively simple feature extraction algorithm. On the basis of extracted signal entropy feature, we can extract the cloud model characteristics of signal entropy feature to build three-dimensional feature of signal .

\section{Cloud Model}

For communication modulation signal recognition, characteristics extraction is largely important. By constructing two-dimensional feature entropy, we can get better signal recognition with smaller computational complexity and less computing time. But it will lead to low recognition rate under low SNR. Therefore, in order to improve recognition rate, we introduce the cloud model theory ${ }^{[12]}$ to get entropy cloud characteristics. At present, the cloud model theory has been widely used in many fields. The ambiguity (the higher the volatility of the numerical range of objects to be described, the more fuzzy of the concept) and the randomness (the randomness of membership of objects to be described) of objects to be described is fully integrated together by this theory to constitute the mutual mapping between qualitative (a concept in discourse domain) and quantitative (the membership degree of the attribute value in discourse domain). It provides a good theoretical basis for the signal feature extraction. 


\subsection{Basic Definition}

The basic definition of the cloud model can be described as follows:

Suppose that there is a common set $X$ (the discourse domain is $X=\{x\}$ ), and fuzzy set $\tilde{A}$ belongs to $X$. For any element $x$, there is a random number $\mu_{\tilde{A}}(x)$ with stable tendency that could be called the affiliation of $x$ to $\tilde{A}$. If all the elements in the discourse domain are simple and orderly, then $X$ can be regarded as basic variables, and the distribution of the membership degree in $X$ is membership cloud. If elements in the discourse domain are not simple and orderly, but $X$ can be mapped to an orderly domain $X^{\prime}$ based on a corresponding rule $f$ and should meet that there is only one $x^{\prime}$ in $X^{\prime}$ corresponding to $x$, then $X^{\prime}$ can be regarded as basic variables, and the distribution of the membership degree in $X^{\prime}$ is membership cloud.

\subsection{Features of Cloud Model}

Cloud model use three numerical features (mathematical expectation $E x$, entropy $E n$ and hyper entropy $\mathrm{He}$ ) to represent the concept as a whole, which can accurately describe signals. Here, we give an example of one-dimensional normal cloud model to illustrate these three characteristics. The example is shown in fig.1, where, $E x=18$, $E n=2, H e=0.2, N=3000$.

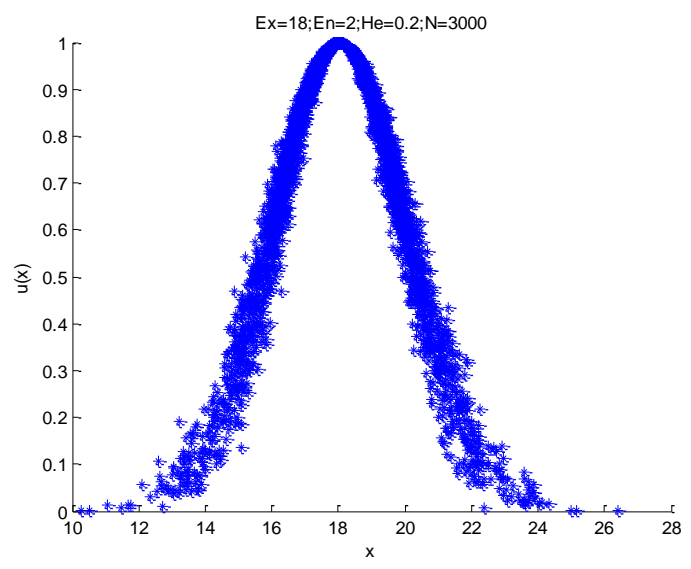

Figure 1. One-Dimensional Normal Cloud Model

From Figure 1, it can be seen that:

Mathematical expectation $E x$ is the numerical expectation of the spatial distribution of cloud droplets, which can best represent the qualitative concept and reflect the gravity center position of cloud droplets group. That is, Ex represents the center of ambiguity concept.

Entropy theory $E n$ is originally used to describe the state parameter of thermodynamic theory, then it is introduced into information theory and statistical physics theory as a measure of the uncertainty degree of objects to be described. In cloud model theory, entropy En represents measurable degree of qualitative concept. The greater the entropy value, the more macro qualitative concept; For another, entropy $E n$ is also an uncertainty measurement of qualitative concept, which is determined by the randomness and fuzziness of the concept. On the one hand, En is random measure of qualitative concept, which reflects discrete degree of cloud droplets; On the other hand, En is fuzziness 
measure of qualitative concept, which reflects value range of cloud droplets. To some extent, $E n$ reveals the relevance between random measure and fuzziness measure.

Hyper entropy $\mathrm{He}$ reflects aggregation of uncertainty of all points in the discourse domain, namely aggregation of cloud droplets. It is an uncertainty measurement of entropy. Its size can be used to express the discrete degree and the thickness of the cloud indirectly, which is determined by the randomness and fuzziness of entropy.

\section{Simulation Results and Analysis}

Here takes six different kinds of modulation signals (AM, FM, PM, ASK, FSK and PSK) as examples to illustrate the superiority of the novel algorithm. We extract the features of signals in different SNR environment, and then use grey correlation classifier for signal identification. The simulation results are shown in fig.2, and the different colors represent the different types of modulated signals.

From fig.2, it can be seen that signal features were extracted by the modulation signal recognition algorithm based on entropy cloud characteristics (three-dimensional feature) have good aggregation in class and separation between classes. With the decrease of SNR, overlapping interval of different signals increase, which brings certain difficulty to subsequent classification recognition. Therefore, we use the grey correlation classifier algorithm to identify and classify those signals with interval features, thus achieves the purpose of signal classification under low SNR.

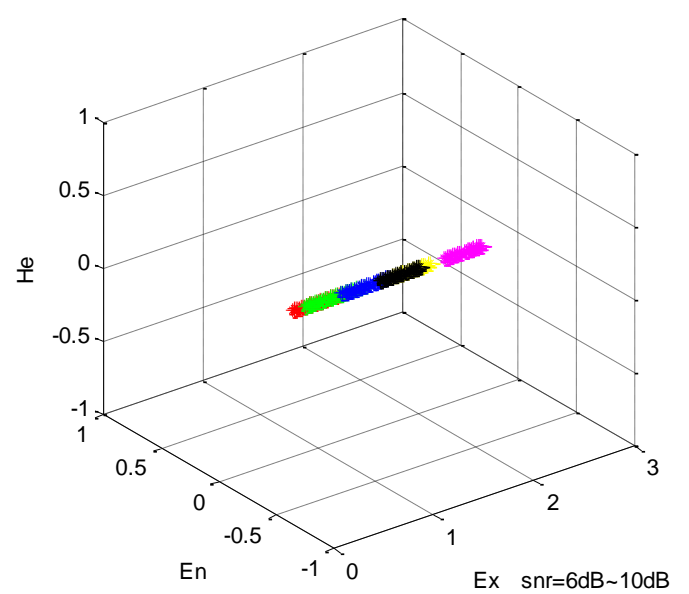

(a) $\mathrm{SNR}=6 \sim 10(\mathrm{~dB})$

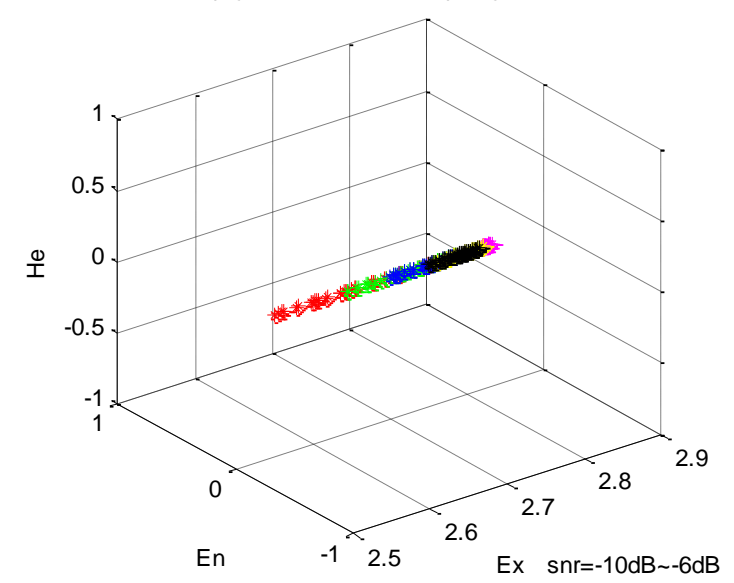

(c) $\mathrm{SNR}=10 \sim-6 \mathrm{~dB}$

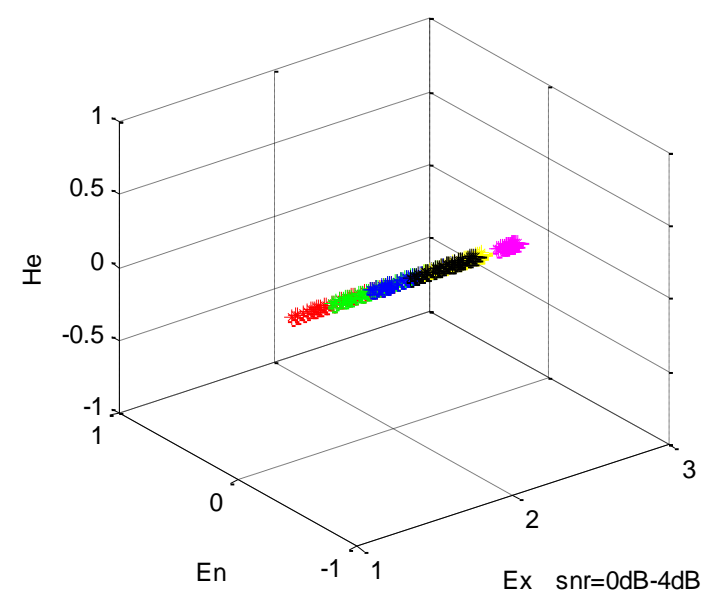

(b) $\mathrm{SNR}=0 \sim 4(\mathrm{~dB})$

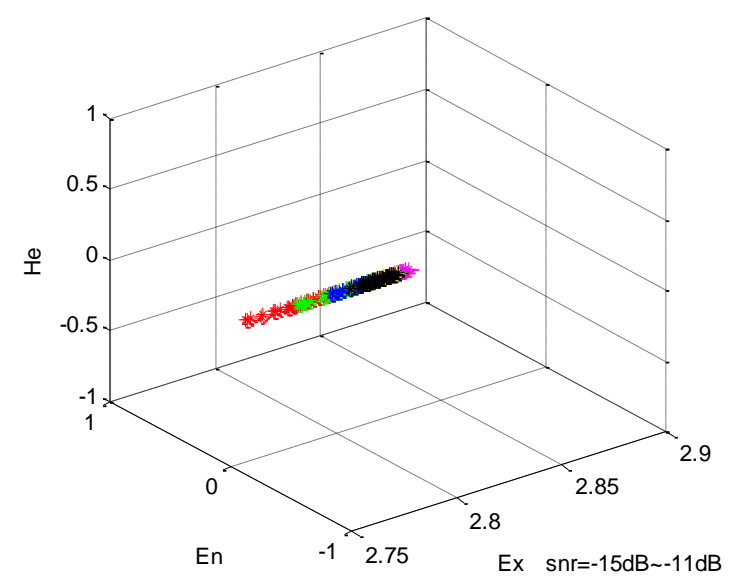

(d) SNR $=-15 \sim-11 \mathrm{~dB}$

Figure 2. Entropy Cloud Features 
We contrast the improved recognition algorithm based on entropy cloud features with the traditional recognition algorithm based on entropy features under different SNRs, and get the recognition rate of various signal, as shown in table.1.

Table 1. Recognition Rate Under Different SNRs by Two Algorithms

\begin{tabular}{|l|l|l|l|l|l|l|}
\hline SNR(dB) & $0 \sim 4$ & $-5 \sim-1$ & $-10 \sim-6$ & $-15 \sim-11$ & $-17 \sim-13$ & $-20 \sim-16$ \\
\hline Entropy features (\%) & 100 & 100 & 97.3 & 65.4 & 42.9 & 38.9 \\
\hline Entropy cloud features (\%) & 100 & 100 & 100 & 97.0 & 81.7 & 76.8 \\
\hline
\end{tabular}

It is clear that the improved recognition algorithm realizes better recognition results under low SNR environment. Even if SNR down to $-15 \sim-11 \mathrm{~dB}$, the recognition rate can still reach 97\%. On the other hand, when SNR changes, the recognition rate of the improved algorithm can remain relatively stable, so it's suitable for practical engineering applications.

\section{Conclusion}

In view of the difficult problem that communication modulation signal is difficult to be identified under low SNR, this paper proposes a new signal feature extraction algorithm. By extracting entropy cloud digital characteristics of the signal, this algorithm describe the distribution characteristics of signal features from different perspectives. Through three-dimensional feature description, signal features can be extracted in unstable SNR environment. Then grey correlation classifier algorithm is used to classify extracted overlapping features for realizing the purpose of communication modulation signal recognition. The simulation results show that the improved algorithm can achieve signal feature extraction and recognition under low and variable SNR. That is, it has good engineering application value.

\section{Acknowledgements}

This paper is funded by the International Exchange Program of Harbin Engineering University for innovation-oriented talents Cultivation, Natural Science Foundation of China (NO. 61301095, NO. 51374099), the Heilongjiang Province Natural Science Foundation(No.F201345) and the Fundamental Research Funds for the Central Universities of China(No.HEUCF150812/150810).

\section{References}

[1] E. E. Azzouz and A. K. Nandi, "Procedure for automatic recognition of analogue and digital modulations.”, IEEE Proceedings on Communications, vol. 143, no. 5, (1996).

[2] A. K. Nandi and E. E. Azzouz, "Algorithms for automatic modulation recognition of communication signals", IEEE Transactions on Communications, vol. 46, no. 4, (1998).

[3] D. Kavalov and V. Kalinin, "Neural network surface acoustic wave RF signal processor for digital modulation recognition", IEEE Transactions on Ultrasonics, Ferroelectrics and Frequency Control. Vol. 49, no. 9, (2002).

[4] L. Wang, and Y. Ren, "Recognition of digital modulation signals based on high order cumulants and support vector machines", ISECS International Colloquium on Computing, Communication, Control, and Management, (2009)

[5] S. Liu, J. Li and C. Zhang, "Research on Electromagnetically Acoustic Emission Signals Using Sample Entropy", 2012 Sixth International Conference on Electromagnetic Field Problems and Applications (ICEF), (2012)

[6] Y. Li, J. Ge and Y. Lin, "Radar emitter signal recognition based on multi-scale wavelet entropy and feature weighting", Journal of Central South University, vol. 21, no. 11, (2014).

[7] W. A. Gardner and C. M. Spooner, "Cyclic spectral analysis for signal detection and modulation recognition”, 21st Century Military Communications Conference, (1988).

[8] X. He, J. Cheng and G. He, "Application of BP neural network and higher order spectrum for shipradiated noise classification", 2010 2nd International Conference on Future Computer and Communication (ICFCC), (2010) 
[9] Y. Li, J. Li and J. Ge, "The application of Improved Generalized Fractal Box-counting Dimension Algorithm in Emitter Signals Recognition", Journal of Information and Computational Science. Vol. 8, no. 14, (2011).

[10] J. Pang , Y. Lin a and X. Xu, "The improved radial source recognition algorithm based on fractal theory and neural network theory", International Journal of Hybrid Information Technology, vol. 7, no. 2, (2014).

[11] X. Tan, H. Zhang and D. Zhu, "Blind Modulation Recognition of PSK Signals Based on Constellation Recovery", Journal of Astronautics, vol. 32, no. 6, (2011).

[12] X. Wei, H. Xie and H. Guo, "An algorithm of digital modulation recognition based on instantaneous characteristic parameters", Transducer and Microsystem Technologies, vol. 30, no. 1, (2011).

[13] L. Bai and N. Yan, "Automatic Modulation Identification for Communication System", Radio Communications Technology, vol. 37, no. 4, (2011).

[14] Z. Zhang, J. Ou and K. HuangFu, "Algorithm for automatic modulation classification of analogue communication signal", Computer Engineering and Science, vol. 35, no. 3, (2013).

[15] X. Wang, "From the entropy and information entropy to the organization.", Modern physics, vol. 15, no. $4,(\mathbf{2 0 0 3})$.

[16] G. Zhang, Y. Sha, Y.Yu. Cloud Classifier Based on Attribute Similarity. Transactions of Beijing Institute of Technology. 25,6.(2005).

[17] M. V. Subbarao, N. S. Khasim, T. Jagadeesh and M. H. H. Sastry, "A novel technique for automatic modulation classification and time-frequency analysis of digitally modulated signals", International Journal of Signal Processing, Image Processing and Pattern Recognition, vol. 6, no. 2, (2013).

[18] Chunjie Zhang, Na Li. Modulation Parameter Estimation and Recognition of PSK signals. Applied Science and Technology. 40,5.(2013).

[19] J. Li, Y. Li and S. Kidera, "A robust signal recognition method for communication system under timevarying SNR environment”, IEICE Transactions on information and systems, vol. 96, no. 12, (2013).

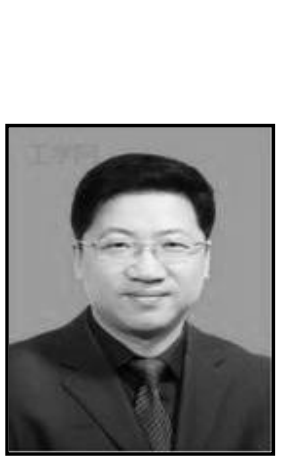

\begin{abstract}
Authors
Yibing $\mathbf{L i}$ received the B.S. and M.S. and Ph.D degrees in Harbin Marine engineering college, Harbin engineering university in 1989, 1997 and 2003, respectively. He has been a teacher in Harbin Engineering University of China since 1989, and became a professor in 2004. During 2007-2008, he stayed at the University of Hong Kong Electronic Engineering lab as a visiting scholar. Now he is a IEEE member, a senior member of China Institute of Communications and a senior member of China Computer Federation.
\end{abstract}

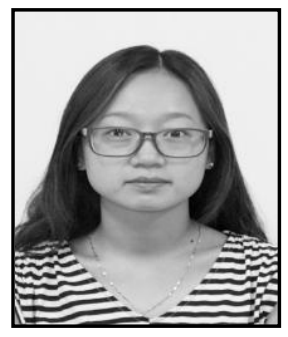

Jie Chen received the B.S. in Electronic Information Engineering from Harbin Engineering University(HEU) in 2014. During 2014-2015, she stayed in HEU to read M.S., and now she is studying Information fusion.

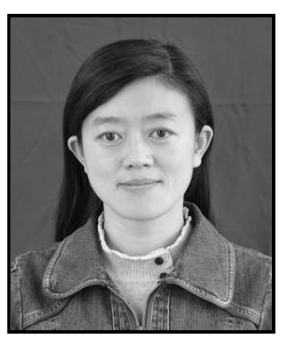

Dandan Liu is a teacher and an associate professor in Heilongjiang University of Science and Technology. Nearly 5 years, she is mainly engaged in the interests as smart material perception and control, multi-resolution signal processing and information fusion. 
Jingchao $\mathbf{L i}$ received the B.S. in Electronic Information Engineering from Harbin Engineering University (HEU) in 2010. During 2010-2011, she stayed in HEU to read M.S., During 2011-2014, she stayed in HEU to read P.D. During 2012-2013, she was in University of Electro-Communication (UEC) as exchanged student. Now she is studying communication signals' recognition. 